\title{
The Importance of the Solids Loading on Confirming the Dielectric Nanosize Dependence of $\mathrm{BaTiO}_{3}$ Powders by Slurry Method
}

\author{
Wei Zhou, Yi Mei Nie, Shu Jing Li, and Hai Yan Liang \\ School of Science, Beijing Technology and Business University, Beijing 100048, China \\ Correspondence should be addressed to Wei Zhou; zhouw@th.btbu.edu.cn
}

Received 7 April 2013; Accepted 27 May 2013

Academic Editors: R. Katiyar and E. Mikuli

Copyright (c) 2013 Wei Zhou et al. This is an open access article distributed under the Creative Commons Attribution License, which permits unrestricted use, distribution, and reproduction in any medium, provided the original work is properly cited.

The dielectric nanosize dependence of $\mathrm{BaTiO}_{3}$ powders was investigated by the slurry method, where two series of $\mathrm{BaTiO}_{3}$ slurries with $10 \mathrm{vol} \%$ and $30 \mathrm{vol} \%$ solids loadings were prepared as model samples. Applying the Bruggeman-Hanai equation, the highfrequency limiting permittivity $\left(\varepsilon_{h}\right)$ of the slurries was extracted from the dielectric spectra. The $\varepsilon_{h}$ of the 10 vol\% slurry showed abnormal size independence in the range from $100 \mathrm{~nm}$ to $700 \mathrm{~nm}$, and the $\varepsilon_{h}$ of the 30 vol\% slurry exhibited good agreement with the previous prediction. Through analysing quantitatively the response of $\varepsilon_{h}$ to the changing permittivity of the powders under different solids loading, it was found that the $\varepsilon_{h}$ of the slurry with lower solids loading is more inclined to be interfered by the systematic and random errors. Furthermore, a high permittivity value was found in the $\mathrm{BaTiO}_{3}$ powders with $50 \mathrm{~nm}$ particle size.

\section{Introduction}

With the achievement of the particle size from several microns downward tens of nanometers, the issue about the nanosize effect of $\mathrm{BaTiO}_{3}$ has attracted intensive attentions [1-3]. However, the reported critical size for $\mathrm{BaTiO}_{3}$ powders spreads out in the wide range between 25 and $110 \mathrm{~nm}[4,5]$. The intrinsic reason is that the grain of $\mathrm{BaTiO}_{3}$ powders would grow up irreversibly during the high temperature sintering. So it is very difficult to obtain the exact critical size of $\mathrm{BaTiO}_{3}$ by means of using traditional solid-state pellet technique. Recently, the dielectric evaluation of ceramic slurries as an in situ method has been of primary interest $[6,7]$. However, the slurry method presented that the permittivity of $\mathrm{BaTiO}_{3}$ powders keeps basically constant when the particle size is above $200 \mathrm{~nm}$ [8]. The result deviates the present theories about the size dependence of $\mathrm{BaTiO}_{3}$ powders and implies that some defects in the slurry method need to be improved. In this paper, we focused on the effect of the solids loading on the slurry method and offered some accurate and valuable information to the dielectric nanosize dependence of $\mathrm{BaTiO}_{3}$ powders.

\section{Experimental}

$\mathrm{BaTiO}_{3}$ powders with seven kinds of particle sizes, 50, 100, $200,300,400,500$, and $700 \mathrm{~nm}$, were commercially obtained from Inframat Advanced Materials, USA. The purities of all powders are higher than $99.95 \%$, and the room-temperature density is $5.85 \mathrm{~g} / \mathrm{cm}^{3}$. $\mathrm{BaTiO}_{3}$ slurries at two solids loadings, $10 \mathrm{vol} \%$ and $30 \mathrm{vol} \%$, were prepared by ultrasonically mixing powders with a propylene carbonate $\left(\varepsilon=66.7\right.$ at $25^{\circ} \mathrm{C}$, from Fisher Scientific) for $30 \mathrm{~min}$. The slurries were then placed in a liquid dielectric test fixture (Agilent Tech., 16452A), and the frequency dependence of the permittivity of the slurries was measured with an impedance analyzer (Agilent Tech. 4294A) from $40 \mathrm{~Hz}$ to $20 \mathrm{MHz}$ at $25^{\circ} \mathrm{C}$.

\section{Result and Discussion}

Figure 1 shows the 3D representations of the particle sizedependent dielectric relaxation spectra of $\mathrm{BaTiO}_{3}$ powders dispersed in propylene carbonate (PC) with $10 \mathrm{vol} \%$ and $30 \mathrm{vol} \%$ solids loadings, respectively. A clear relaxation behavior can be seen at near $10^{4} \mathrm{~Hz}$, which is aroused from 


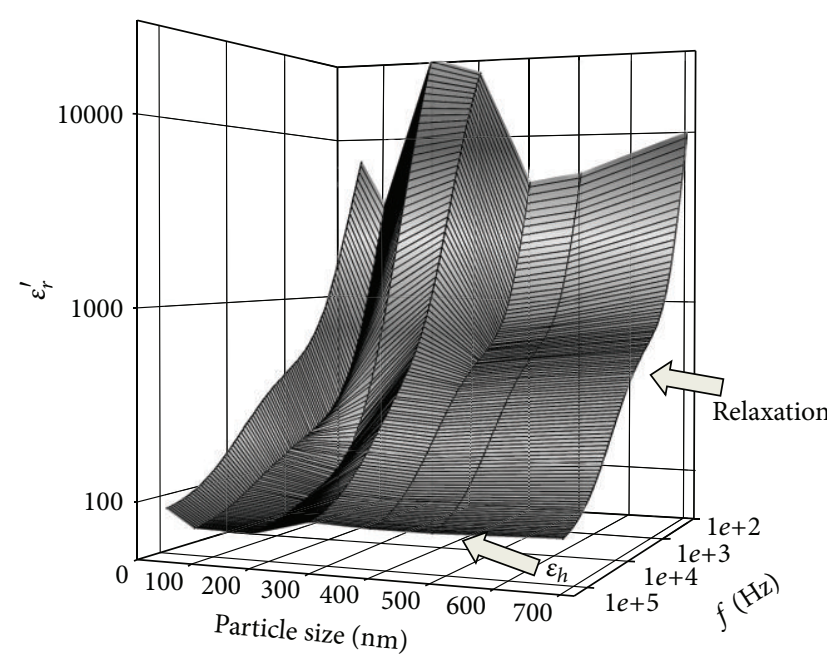

(a)

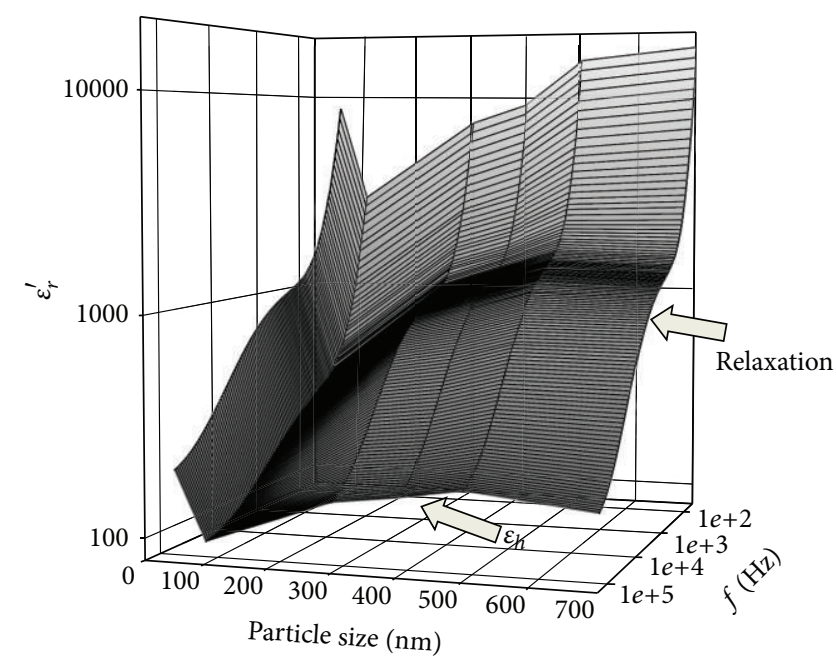

(b)

FIGURE 1: Three-dimensional representations of the nanosize dependence of the permittivity spectra of the $\mathrm{BaTiO}_{3}$ slurries with two solids loadings (a) 10 vol\% and (b) 30 vol\%.

the interfacial polarization between the $\mathrm{BaTiO}_{3}$ powders and the PC. By using Bruggeman-Hanai equation [9]

$$
\frac{\varepsilon_{h}-\varepsilon_{p}}{\varepsilon_{m}-\varepsilon_{p}}\left(\frac{\varepsilon_{m}}{\varepsilon_{h}}\right)^{1 / 3}=1-\varphi,
$$

the permittivity of $\mathrm{BaTiO}_{3}$ powders $\left(\varepsilon_{p}\right)$ can be calculated directly from the high-frequency limiting permittivity of the slurry $\left(\varepsilon_{h}\right)$ in the case that the volume fraction of the powders in the slurry $(\varphi)$ and the permittivity of the PC $\left(\varepsilon_{m}\right)$ are known accurately. So $\varepsilon_{h}$ is a significant dielectric parameter in order to obtain the $\varepsilon_{p}$ with small margin of error.

Figure 2(a) shows the particle size dependence of $\varepsilon_{h}$ of $\mathrm{BaTiO}_{3}$ slurries with different $\varphi$. According to the nonlinear relationship between $\varepsilon_{h}$ and $\varphi$ in (1), the $\varepsilon_{h}$ will increase with increasing $\varphi$ if the $\varepsilon_{p}$ is larger than the $\varepsilon_{m}$. The permittivity of the $\mathrm{PC}$ is about 67 at room temperature, which is much smaller than the permittivity of $\mathrm{BaTiO}_{3}$ powders $(1000 \sim 5000)$. So it is reasonable that the $\varepsilon_{h}$ of $30 \mathrm{vol} \% \mathrm{BaTiO}_{3}$ slurry is much higher than the one of 10 vol\% slurry at each particle size. Furthermore, the $\mathrm{BaTiO}_{3}$ slurries with different volume fractions display distinct particle size dependence. For the slurry with 10 vol\% solids loading, $\varepsilon_{h}$ keeps constant basically on the size range from $700 \mathrm{~nm}$ to $300 \mathrm{~nm}$, which agrees with the results in Wada's research [8]. For the slurry with 30 vol\% solids loading, however, the $\varepsilon_{h}$ displays a nonlinear size dependence similar with the results from solids method [10]. The difference implies that the analysis of the solids loading might explain the discrepancy between the previous results from the slurry and pellet methods.

Now, the permittivity of the $\mathrm{BaTiO}_{3}$ powders is supposed to decrease from 4000 to 3000 with decreasing particle size, and the permittivity of the $\mathrm{BTiO}_{3}$ slurries with different given solids loading is calculated by (1). According to the results listed in Table 1, $\varphi$ dependence of change rate in $\varepsilon_{h}\left(\Delta \varepsilon_{h} \%=\right.$ $\left.\left(\varepsilon_{h, 2}-\varepsilon_{h, 1}\right) / \varepsilon_{h, 2} \times 100 \%\right)$ can be found. With the volume fraction becoming diluted from $25 \%$ to $4 \%$, the $\Delta \varepsilon_{h} \%$ decreases
TABLE 1: The response sensitivity of $\varepsilon_{h}$ to the $\varepsilon_{p}$ under different solids loadings.

\begin{tabular}{lccc}
\hline Given $\varphi$ & $\varepsilon_{h, 1}$ & $\varepsilon_{h, 2}$ & $\Delta \varepsilon_{h} \%$ \\
\hline $4 \%$ & 74.547 & 74.695 & 0.20 \\
$10 \%$ & 89.127 & 89.622 & 0.56 \\
$17 \%$ & 111.08 & 112.29 & 1.09 \\
$25 \%$ & 145.27 & 148.03 & 1.90 \\
\hline
\end{tabular}

gradually from $1.90 \%$ to $0.20 \%$. It means that the diluter the slurry is, the higher the requirement to the measurement accuracy will be. In experimental measurements, there are always both systematic and random errors, which are mixed into the original data usually. For the dilute slurry, the small $\Delta \varepsilon_{h} \%$ is more inclined to be interfered by the systematic and random errors and offers inaccurate information. So the solids loading of the slurry should be increased high enough in order to weaken the confused influence of the systemic error on the original data.

Considering the positive effect of higher solids loading on the accuracy of the slurry method, the $\varepsilon_{h}$ from Figure 1(b) was assigned into (1) for calculating the permittivity of $\mathrm{BaTiO}_{3}$ powders, $\varepsilon_{p}$. Figure $2(\mathrm{~b})$ shows the size dependence of $\varepsilon_{p}$, which has the similar size dependence with the corresponding $\varepsilon_{h}$. It can be found that the $\varepsilon_{p}$ increased obviously with the particle size decreasing from $700 \mathrm{~nm}$ to $500 \mathrm{~nm}$, and then decreased gradually with the particle size decreasing from $500 \mathrm{~nm}$ to $100 \mathrm{~nm}$. The result agrees with the current postulation from the pellet method. The $\varepsilon_{p}$ dependence in fine $\mathrm{BaTiO}_{3}$ structures is driven by a combined effect of the ferroelectric core whose nonlinearity is gradually reducing, together with the increasing amount of the nonferroelectric "dead-layer" grain boundaries, when reducing the grain size down to tenths of nanometers [1]. However, the $\varepsilon_{p}$ with $50 \mathrm{~nm}$ 


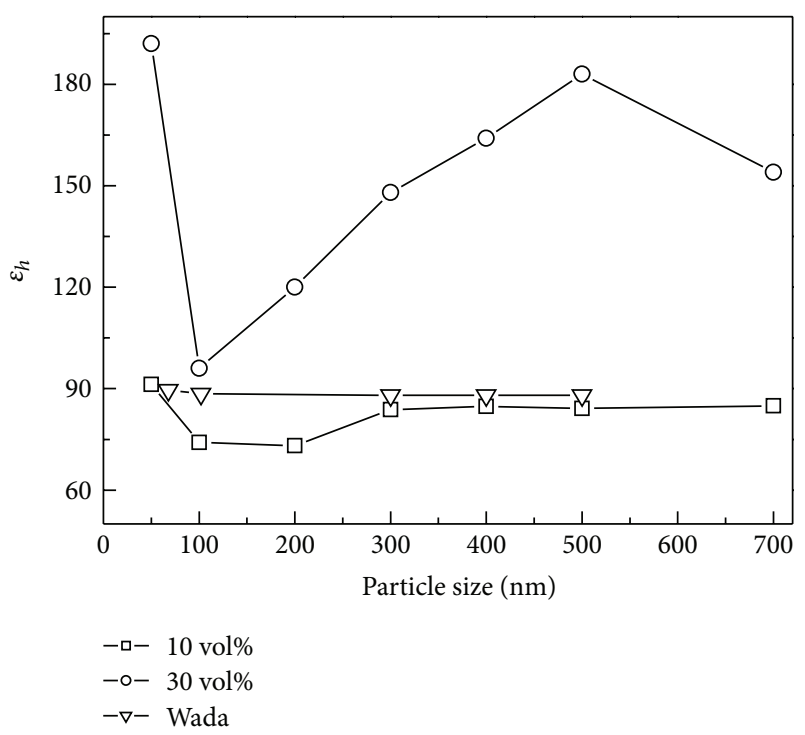

(a)

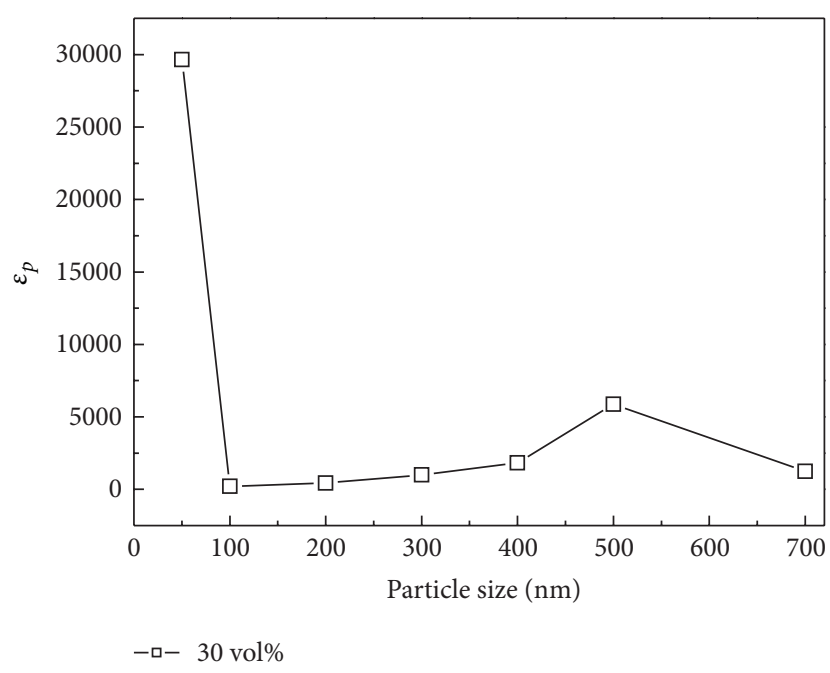

(b)

FigurE 2: The size dependence of the relative dielectric parameters of the $\mathrm{BaTiO}_{3}$ slurries.

particle size appeared with significant high value, which was reported only in Wada's slurries method. There is not enough attention for explaining the phenomenon yet, and we will focus on it in our following research.

\section{Conclusions}

The importance of the solids loading in the accuracy of the sully method was researched in order to get the accurate nanosize effect of $\mathrm{BaTiO}_{3}$ powders. By means of comparing the dielectric data and calculation of the $\mathrm{BaTiO}_{3}$ slurries with two different solids loadings, $10 \mathrm{vol} \%$ and $30 \mathrm{vol} \%$, the higher one was found to possess lower calculation error. Based on the finding, the conflicting nanosize dependence of the $\mathrm{BaTiO}_{3}$ powders obtained from previous slurry and pellet method was attributed to the low solid loading used in the slurry method.

\section{Acknowledgments}

The work was supported by the National Natural Science Foundation of China (no. 21003005) and the Scientific Research Foundation for the Returned Overseas Chinese Scholars, State Education Ministry (SRF for ROSC, SEM). The authors acknowledge Juan C. Nino and Hyuksu Han for helpful discussions.

\section{References}

[1] L. Curecheriu, M. T. Buscaglia, V. Buscaglia, Z. Zhao, and L. Mitoseriu, "Grain size effect on the nonlinear dielectric properties of barium titanate ceramics," Applied Physics Letters, vol. 97, no. 24, Article ID 242909, 2010.

[2] P. Sedykh, D. Michel, E. V. Charnaya, and J. Haase, "Size effects in fine barium titanate particles," Ferroelectrics, vol. 400, no. 1, pp. 135-143, 2010.
[3] C. Fang, D. Zhou, and S. Gong, "Core-shell structure and size effect in barium titanate nanoparticle," Physica B, vol. 406, no. 6-7, pp. 1317-1322, 2011.

[4] J. Petzelt, "Dielectric grain-size effect in high-permittivity ceramics," Ferroelectrics, vol. 400, no. 1, pp. 117-134, 2010.

[5] J. H. Qiu, J. N. Ding, N. Y. Yuan, H. X. Cao, X. Q. Wang, and Y. Zhou, "Phase diagram and ferroelectric behaviors of $\mathrm{BaTiO}_{3}$ nanowires," Journal of Applied Physics, vol. 109, no. 5, Article ID 054103, 2011.

[6] V. Petrovsky and F. Dogan, "Fundamentals of impedance spectral analysis applied to determine permittivity of dielectric particles," Journal of the American Ceramic Society, vol. 92, no. 5, pp. 1054-1058, 2009.

[7] S. Wada, T. Hoshino, and H. Yasuno, "Dielectric properties of $\mathrm{nm}$-sized barium titanate fine particles and their size dependence," Advances in Electronic Ceramic Materials: Ceramic Engineering and Science Proceedings, vol. 26, pp. 89-100, 2005.

[8] S. Wada, H. Yasuno, T. Hoshina, S.-M. Nam, H. Kakemoto, and T. Tsurumi, "Preparation of nm-sized barium titanate fine particles and their powder dielectric properties," Japanese Journal of Applied Physics, vol. 42, no. 9, pp. 6188-6195, 2003.

[9] W. Zhou, B. B. Hinojosa, and J. C. Nino, "Applicability of the bruggeman equation for analyzing dielectric slurries containing ceramic powders with high permittivity," Journal of the American Ceramic Society, vol. 95, no. 2, pp. 457-460, 2012.

[10] M. H. Frey, Z. Xu, P. Han, and D. A. Payne, "The role of interfaces on an apparent grain size effect on the dielectric properties for ferroelectric barium titanate ceramics," Ferroelectrics, vol. 206, pp. 337-353, 1998. 

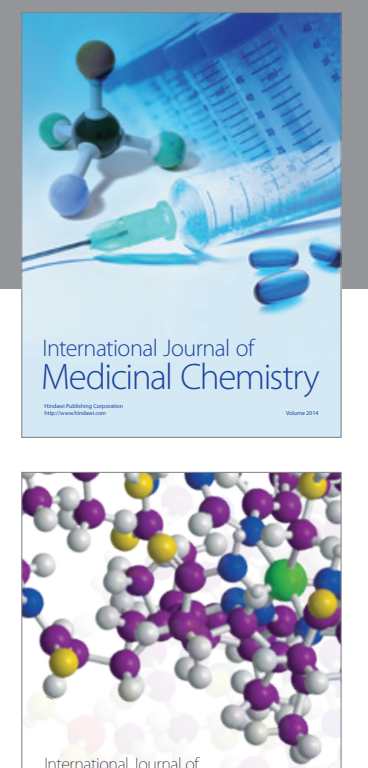

\section{Carbohydrate} Chemistry

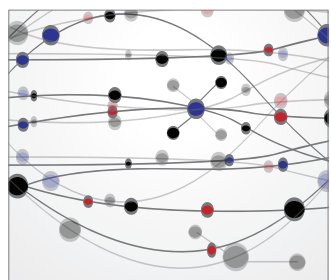

The Scientific World Journal
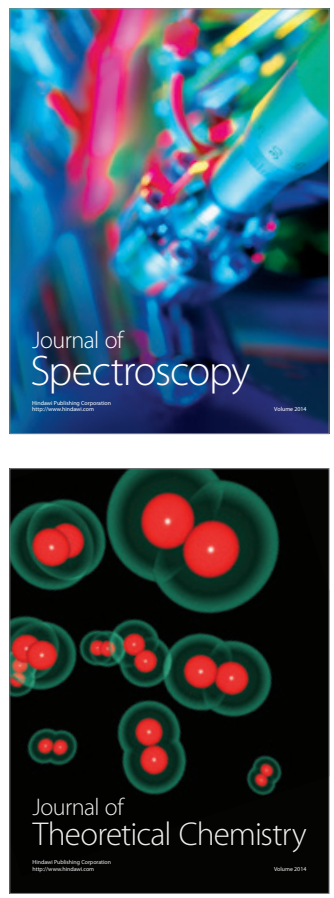
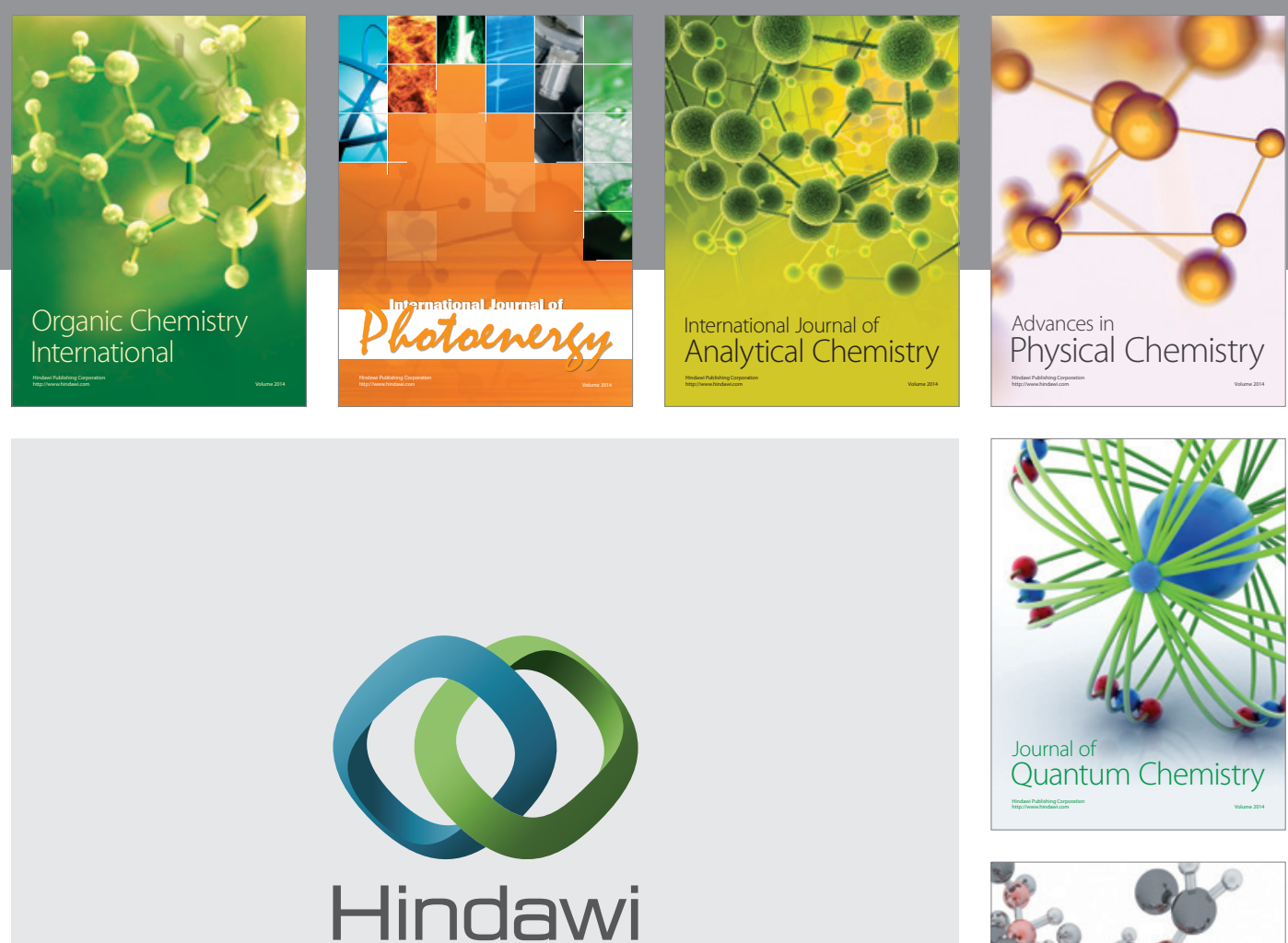

Submit your manuscripts at

http://www.hindawi.com

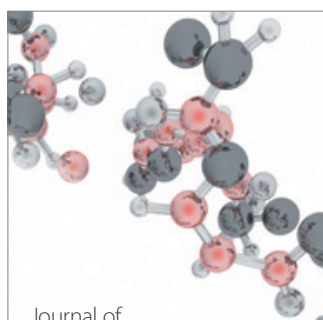

Analytical Methods

in Chemistry

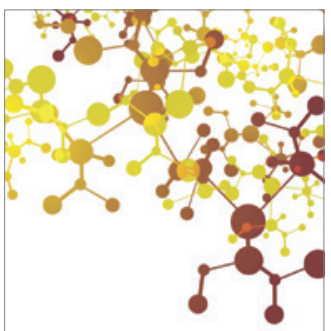

Journal of

Applied Chemistry

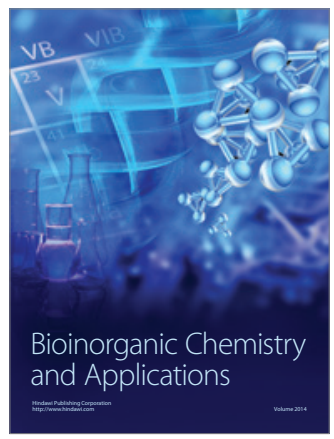

Inorganic Chemistry
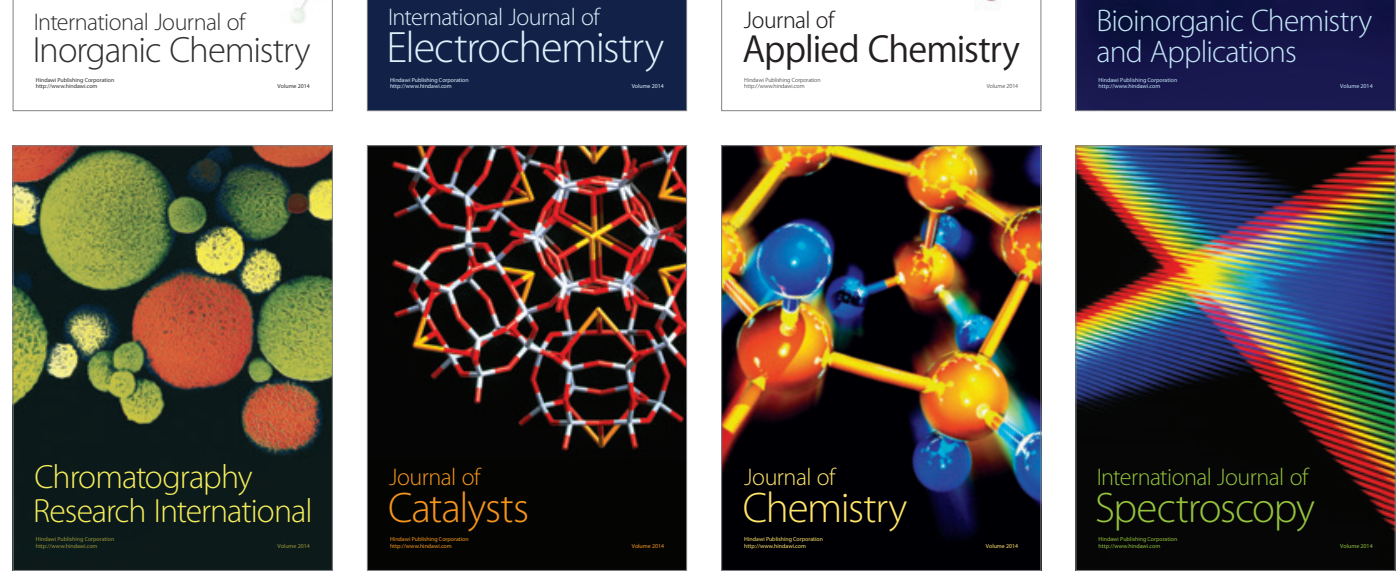